Research Article

\title{
Predicting Mental Health Problems with Automatic Identification of Metaphors
}

\author{
Nan Shi, ${ }^{1}$ Dongyu Zhang $\mathbb{D}^{1}{ }^{1} \mathrm{Lulu} \mathrm{Li}^{1}$ and Shengjun $\mathrm{Xu}{ }^{2}$ \\ ${ }^{1}$ School of Software, Dalian University of Technology, Dalian 116620, China \\ ${ }^{2}$ Psychological Health Education and Counseling Center, Dalian University of Technology, Dalian 116620, China \\ Correspondence should be addressed to Dongyu Zhang; zhangdongyu@dlut.edu.cn
}

Received 19 February 2021; Revised 18 March 2021; Accepted 10 April 2021; Published 30 April 2021

Academic Editor: Han Wang

Copyright (C) 2021 Nan Shi et al. This is an open access article distributed under the Creative Commons Attribution License, which permits unrestricted use, distribution, and reproduction in any medium, provided the original work is properly cited.

Mental health problems are prevalent and an important issue in medicine. However, clinical diagnosis of mental health problems is costly, time-consuming, and often significantly delayed, which highlights the need for novel methods to identify them. Previous psycholinguistic and psychiatry research has suggested that the use of metaphors in texts is linked to the mental health status of the authors. In this paper, we propose a method for automatically detecting metaphors in texts to predict various mental health problems, specifically anxiety, depression, inferiority, sensitivity, social phobias, and obsession. We perform experiments on a composition dataset collected from second-language students and on the eRisk2017 dataset collected from Social Media. The experimental results show that our approach can help predict mental health problems in authors of written texts, and our algorithm performs better than other state-of-the-art methods. In addition, we report that the use of metaphors even in nonnative languages can be indicative of various mental health problems.

\section{Introduction}

Mental health problems have become increasingly serious. They not only endanger people's physical and mental health, but also affect the development of the country and society. The WHO survey (https://www.who.int/health-topics/mentalhealth) shows that about $13 \%$ of people worldwide suffer from mental disorder, which cost the global economy one trillion dollars each year. Depression is one of the main causes of disability. Suicide is the second leading cause of death among children aged 15-29. About $20 \%$ of children and adolescents in the world suffer from mental illness, and the highly educated population also suffer from psychological distress which affects their academic performance [1-3]. However, clinical diagnosis of mental health problems is costly, time-consuming, and often significantly delayed, which highlights the need for novel methods to identify these conditions.

Metaphorical expressions are frequently used in human language [4-7]. They involve both linguistic expression and cognitive processes [8] and are an implicit way to convey emotions [9-11]. Human emotions and mental state, which are important for mental health, are frequently communicated and expressed through metaphors. This suggests that the use of metaphorical expressions in texts may indicate mental and cognitive status and so help in mental health screening.

Psycholinguistic and psychiatry studies have indicated that the use of metaphors in texts is linked to the mental health illness of their authors [12-16]. For example, patients with schizophrenia may metaphorically use the phrase "time vessels" to refer to watches and "hand shoes" to refer to gloves. In other words, the use of metaphor in individuals with mental illness may differ from those without, which could offer new opportunities to identify mental illness using metaphors as a diagnostic indicator. Although it is not clear what causes these deviations in metaphor production, neuroscience research offers some clues. Scholars note that some mental illnesses such as schizophrenia relate to dysfunction of the amygdala, which processes and regulates emotion [17]. Other research suggests that metaphorical texts are associated more with activation of the amygdala than with areas relating to literal speech [18]. 
With the development of artificial intelligence and various data processing technologies [19-25], the efficiency of modern medical diagnosis is constantly improving. As an important part of artificial intelligence, natural language processing is widely applied in mental health related issues [26-28]. Shatte et al. [29] reviewed the application of machine learning in mental health: four main application areas, including detection and diagnosis [30, 31]; prognosis, treatment, and support; public health; research and clinical management. The most common mental health conditions addressed include depression, schizophrenia, and Alzheimer's disease. Prior work has shown the feasibility of using NLP techniques with various features extracted from text messages such as linguistic, demographic, and behavioral features to predict mental illness such as depression [32], suicidality [33], and posttraumatic stress disorder [34]. However, few studies have involved the application of metaphor, a deep semantic feature, as a means of detecting and predicting mental health problems. Along with the rapid explosion of social media applications such as Twitter and Facebook, there seems to be a significant increase in metaphorical texts on a variety of topics, including products, services, public events, and tidbits of people's lives. It seems to be an important and promising challenge to leverage metaphor features for supporting the identification and prediction of mental health problems.

In this paper, we propose the use of automatically detected metaphors in texts to predict various mental health problems including anxiety, depression, inferiority, sensitivity, social phobias, and obsession. We named our method MetaphorSentiment Model (MSM) and we performed experiments on a compositional dataset we created from second-language student essays and the eRisk2017 dataset collected from Social Media. Our contributions are as follows.

(i) We propose a novel approach to identify several mental health problems by using linguistic metaphors in texts as features. To the best of our knowledge, we are the first to leverage metaphor features for supporting the identification and prediction of mental health problems.

(ii) The experimental results show that our proposed approach can help predict the mental health of authors of written texts and our algorithm gives fairly good performance, compared to state-of-theart methods.

(iii) The work shows how semantic content, specifically usage of metaphors in writings produced by individuals, can help in detection of six mental health problems. This seems to be a new result where usage of metaphors even in nonnative languages can be used as indicative of various mental health problems.

(iv) We contribute to a novel, scarce, and valuable dataset, which will be released publicly, consisting of second-language speakers' essays and data on authors' mental health problems obtained from a psychological survey. (v) Due to the scarcity of relevant work, exploring features that influence mental health using computational approaches can potentially help with early detection and treatment of mental health and related problems.

\section{Related Work}

2.1. Mental Health in NLP. NLP techniques have been applied to speculate on people's mental health status, based on written texts, such as those on Facebook, Twitter, etc., and they can be used to obtain information on the user's psychological state directly and efficiently [35]. In recent years, scholars have explored many different features of various datasets to explore the mental health status that lies behind a text. Nguyen et al. [36] used data from the foreign Live Journal Post website to collect $38 \mathrm{k}$ posts from the mental illness community and $230 \mathrm{k}$ posts from mentally healthy communities for mental illness prediction. They tried various approaches, including linguistic inquiry and word count (LIWC), which obtain features of language, social, effective, cognitive, perceptual, biological, relativistic, personal attention, and oral, emotional feature (also based on LIWC) and latest dirichlet allocation (LDA) topic models, ultimately achieving 93\% accuracy. Franco-Penya and Sanchez [37] built a tree structure based on the n-grams feature and combined other features and support vector machine (SVM) learning methods to design classifiers to detect mental health status in CLPsych2016 [38]. Cohan et al. [39] comprehensively considered lexical features, contextual features, textual data features, and textual topical features on the same dataset, using SVM classifiers to complete detection tasks. Ramiandrisoa et al. [40] tried a variety of lexical features in another evaluation task on the CLEF 2018 eRisk database [41], including bag of word models, specific category words, and special word combinations, and they converted text into vectors for classification. Weerasinghe et al. [42] investigated language patterns that differentiate individuals with mental illnesses from a control group, including bag-of-words, word clusters, part of speech n-gram features, and topic models to understand the machine learning model.

In addition to the use of text and other user characteristics, the rise of deep learning has provided new ways to detect mental illness through text. Benton et al. [43] modeled multiple scenarios to predict different suicide risk levels and built a multitasking learning framework (MTL) to meet the needs of different tasks. Trotzek et al. [44] first converted text into vectors and then completed classification task through a convolutional neural network to predict the mental health status of the user. Sekulic and Strube [45] applied a hierarchical attention network and analyzed phrases relevant with social media users' mental status by inspecting the model's word-level attention weights. Multimodal thinking is also applied in mental health research $[46,47]$. They used a multimodal approach that consists of jointly analyzing text and visual and audio data and their relation to mental health more than text analysis. 
2.2. Datasets. As discussed above, metaphorical expressions are associated with mental and cognitive status. Since a metaphor involves cognitive processes, it may be feasible to screen and monitor mental and affective status no matter the degree of fluency in the language. We thus assume that metaphor is an important textual feature for mental health detection among language users, including both native and second-language speakers. We collected data from two different sources to verify our assumptions and increase the reliability of our experiments in this study of the relationship between metaphor use and mental health status.

\subsection{Student Composition and Mental Health. We collected} English composition data from English-proficient Chinese college students who speak English as a second language. We also collected mental health data from these students using a psychological survey. First, we used online and offline campus advertisements to recruit 164 college freshman participants who passed the national college students' English level 4 test in China, which means they are native Chinese and fluent in English writing. Prior to participation, all participants provided a consent form indicating their willingness to take part in the study. Participants provided their personal information via a questionnaire and then wrote a composition with 500 English words or more within a two-hour period. The composition had two parts: described their previous life experience and then presented their future plans, including their ideal future lives, thoughts on life, targets for their future lives, and plans to overcome barriers. The content gave us a deep understanding of their psychological states [48], which is essential for the detection of mental health problems.

After writing their composition, students were required to complete a mental health questionnaire that assessed two levels of mental health problems. The first level involved is serious mental health problems, mainly serious psychoses such as hallucinations, suicidal behavior, and suicidal inclination. In our survey, only a few students had first-level problems. The second level involved common mental problems, such as anxiety, depression, inferiority, sensitivity, and social phobias. Mental problems were assessed on the basis of the standard score for screening indexes. Specifically, participants were assessed with mental health problems when their scores on certain indexes exceeded typical results. We excluded data from 8 students because we could not match their mental problems with fuzzy indexes from their mental health data. Effective mental health data for the remaining 156 students is presented in Table 1. Meanwhile, we extracted data from students without mental health problems to use as controls for analyzing differences in metaphor use with sentiment features in texts.

The process of data collection lasted four months and resulted in a total of 156 compositions with 130,044 words from 156 students (aged $18-23 \mathrm{yrs}$, mean $=19.06 \mathrm{yrs}$, $\mathrm{SD}=0.19$, males $=86$, and females $=70$ ), together with mental health data obtained from the psychological questionnaire. These data were kept secure and stored with no identifying factors, i.e., consent forms and questionnaires.
TABLE 1: Mental health data of students.

\begin{tabular}{lccc}
\hline Problem & No. of students & Problem & No. of students \\
\hline Anxiety & 36 & Sensitivity & 49 \\
Depression & 36 & Social phobia & 44 \\
Inferiority & 29 & Obsession & 38 \\
One problem & 28 & Two problems & 21 \\
Three problems & 21 & Four & 10 \\
Five problems & 7 & problems & 7 \\
\hline
\end{tabular}

$N$ problems mean the students with $n$ mental problems at the same time.

2.4. eRisk2017 Data. The eRisk2017 task on early risk detection of depression [49] provides a dataset containing posting contents and comments from Reddit. The task identified 135 Reddit users with depression and 752 Reddit users without depression through their posts and comments. The word quantity for each Reddit user varies from 10 to 2,000. The dataset for each Reddit user contains individual identification, writing data, text title, writing type, and writing contents. Paper [50] details the construction of the eRisk data. They first selected Reddit from multiple social media and collected the post of depression diagnosis through specific search (such as I was diagnosed with depression). Posts are manually evaluated to identify users who are really diagnosed with depression. They collected patients' text records published on Reddit over a period of time. We combined the contents for each Reddit user in chronological order for the present study.

\section{Methodology}

Our work flow is shown in Figure 1. Metaphor is linked to the mental health problems as described above. We extracted metaphors from texts and designed metaphor feature sets to predict various mental health problems. Our method also considered sentiment features in the sample texts as this feature has been widely used in mental health research $[14,44,51,52]$. We applied metaphor and sentiment features in our Metaphor-Sentiment Model (MSM) to predict mental health problem. The feature extraction algorithm is briefly summarized in algorithm 1, and more details will be introduced below.

3.1. Metaphor Feature Extraction. For metaphor-based features, we considered the following (Algorithm 1, Step 1):

(i) The percentage of tokens tagged as metaphor by the automatic metaphor identification method

(ii) The probability of a sentence containing metaphor

We also considered the sentiment of metaphor expressed in a sentence that is consistent with the sentence sentiment. First, SentiStrength (http://sentistrength.wlv.ac.uk/) was used to analyze the overall sentiment of a sentence. SentiStrength analysis yields two scores for sentiment strength: negative (scores -1 to -5 ) and positive (scores 1 to 5 ). The sum of the two values is the overall sentiment score for the sentence. A sentiment score of 0 is defined as neutral. Next, 


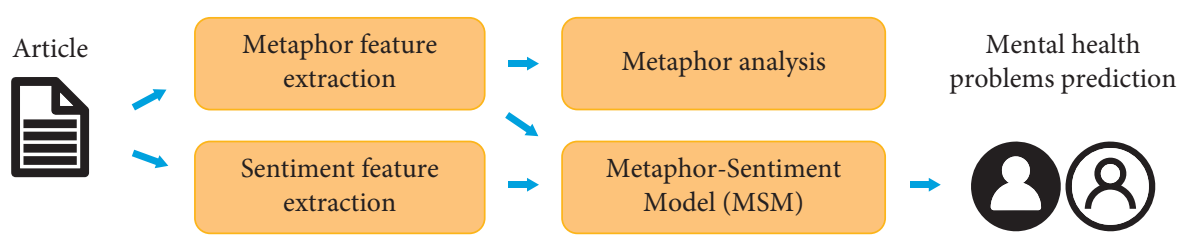

FIgURE 1: The figure of our work flow.

we determined sentiment of metaphor using three specific feature values (Algorithm 1, Step 3):

(i) The number of metaphors with positive sentiment (positive sentiment score)

(ii) The number of metaphors with negative sentiment (negative sentiment score)

(iii) The average sentiment score for all metaphors

In our method, metaphors were identified automatically using a technique that has shown the best performance for token-level metaphor identification tasks to date [53]. The automatic metaphor identification system contains four steps: (1) it trains word embeddings on a Wikipedia dump based on Continuous Bag of Words (CBOW) and SkipGram models to obtain input and output vectors for every word; (2) it selects detected words to assess metaphoricity and separates the detected words from a given sentence; (3) it extracts all possible synonyms and direct hypernyms, including their inflections, of the detected word from WordNet, and it adds them to the candidate word set $w$, which contains all possible senses of the detected word; and (4) it selects the best fit word $w^{*}$, which represents the actual sense of the detected word in the a given sentence, from the candidate word set $w$, using the following formula:

$$
w^{*}=\arg \max _{k} \operatorname{SIM}\left(v_{k}, v_{\text {context }}\right),
$$

where $k \in w, v_{k}$ is the input vector of the CBOW or SkipGram entry for a candidate word $k$, and $v_{\text {context }}$ means the average of all input vectors for context words. The best fit word has the highest cosine similarity with the context words. Finally it computes the similarity value for the detected word and the best fit word using output vectors to measure the difference of sense between the detected word and the context. The detected word is labeled as metaphorical when the similarity value is less than the given threshold. In practical applications we detected every content word in the sentence. The detailed process is presented in Algorithm 2.

We trained and tested the identification algorithm on a metaphor dataset developed by Mohammad [10] that contains 210 metaphorical sentences whose detected words are annotated manually with at least $70 \%$ agreement. We selected the same number of literal sentences from thousands of literal sentences in the dataset. The best metaphor identification performance had a precision of 0.635 , recall of 0.821 , and F1 value of 0.716 with a threshold of 0.5 , which matches the identification performance reported by Mao [53].
For evaluating the performance of the metaphor identification method with our dataset, we randomly selected ten compositions from each of the seven groups that correspond to six mental health problems and healthy control. In total, seventy compositions were analyzed. The metaphor identification performance using the student dataset had a precision of 0.632 , recall of 0.935 , and F1 value of 0.754 .

Figure 2 shows examples of metaphors detected by the automatic metaphor identification method from the student composition dataset (a-c) and eRisk2017 dataset (d-f). The sentences match two words from different domains: for example, a source word tagged as metaphorical, such as broken, and a target word such as dream. However, this token-level metaphor identification algorithm produces some errors since it identifies a metaphor based on local information around the detected word and cannot effectively recognize fixed collocation. For example, in the phrase I ultimately got up on my own, the algorithm mistakenly tags the word own in on my own as metaphorical.

3.2. Sentiment Feature Extraction. The sentiment feature set included the average value of the five dimensions of all words; the proportion of positive sentences, negative sentences, and neutral sentences; the average emotional score of the sentences, and the emotional fluctuation value of each article, yielding ten specific features in total.

We used SentiStrength to obtain sentiment scores for sentences in the texts as above, in order to calculate the proportion of positive sentence, negative sentence, and neutral sentence; the average sentiment score of the sentences of article; the sentiment fluctuation score of each article (Algorithm 1, Step 2).

The average score of the sentences in each article was calculated to determine the emotional value of the article using the following formula:

$$
E=\frac{\sum_{i=1}^{n} S_{i}}{n},
$$

where $E$ represents the average sentiment value of the text, $S_{i}$ represents the emotional score of the $i$ th sentence, and $n$ is the number of the sentences in the text. And the fluctuation score is obtained by subtracting the emotional scores of two consecutive sentences in the article and taking the absolute value. We used the average as its sentiment fluctuation value. It is determined by the following formula:

$$
F=\frac{\sum_{i=2}^{n}\left|S_{i}-S_{i-1}\right|}{(n-1)}
$$


Input: The target text.

Output: Metaphor-Sentiment feature set.

(1) Identifying the metaphoricity of each word in the text, count the frequency, and generate metaphorical statistical features

(2) Using Sentistrength to obtain the score of positive and negative emotions, and generate the statistical characteristics and sentiment uctuation value on sentence level

(3) Determining the metaphorical words in the sentence by the sentiment information of the sentence to obtain the sentiment characteristics of the metaphorical words

(4) Using SenticNet to get the word-level emotional scores of five dimensions, and calculating the average value to get the sentiment features of the text

(5) Integrating the above characteristics, return Metaphor-Sentiment feature set

Algorithm 1: Framework of feature set generation for MSM.

Input: sentence; A dictionary that returns the corresponding word vector of key, Word2vec; A dictionary that returns the related word set of key, WordNet.

Output: A list of the corresponding metaphority labels of words in sentence, labels.

(1) function TokenMetaphorIdentify (detected word; sentence)

(2) $\quad$ context $=[] ; w^{*}=0$

(3) $\quad w=$ WordNet $[$ detected word $]$

(4) for each word $\in$ sentence and word $\neq$ detected_word do

(5) $\quad$ context $=$ context $\cup$ word

(6) end for

(7) $\quad v_{\text {context }}=$ average $($ Word2vec $[$ context $])$

(8) for each $\mathrm{k} \in w$ do

(9) if cosine $\left(W o r d 2 v e c[k], v_{\text {context }}\right)>$ max_cosine then

(10) max_cosine $=\operatorname{cosine}\left(\right.$ Word $\left.2 v e c[k], v_{\text {context }}\right)$

(11) $\quad w^{*}=k$

(12) end if

(13) end for

(14) metaphor_value $=$ cosine $\left(\right.$ Word2vec $\left[\right.$ detected_word], Word $\left.2 v e c\left[w^{*}\right]\right)$

(15) if metaphor value $<$ threshold then

(16) return True

(17) else

(18) return False

(19) end if

(20) end function

(21)

(22) function MetaphorIdentify (sentence)

(23) labels $=$ []

(24) for each word $\in$ sentence do

(25) label $=$ TokenMetaphorIdentify (word; sentence)

(26) labels = labels $\cup$ label

(27) end for

(28) return labels

(29) end function

Algorithm 2: Metaphor identification algorithm.

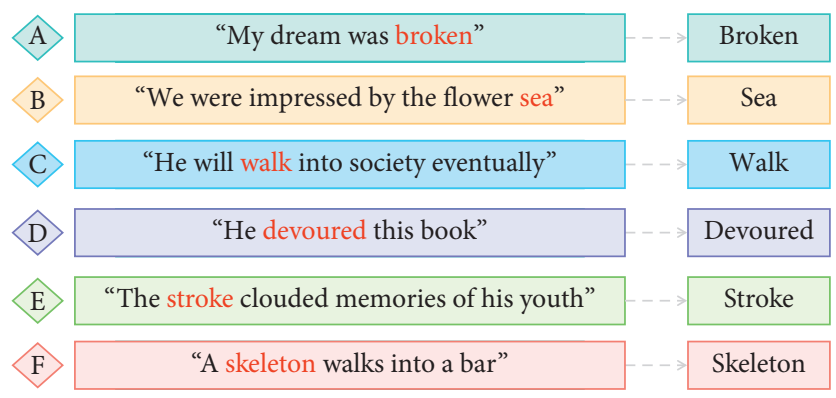

FIGURE 2: Metaphor examples identified by automatic metaphor identification method. 
where $F$ represents the emotional fluctuation value of the text.

Scores for the five dimensions (values of pleasantness, attention, sensitivity, aptitude, and polarity) were obtained using SenticNet (http://www.sentic.net) (Algorithm 1, Step 4). The averages of the five dimensions of all words were taken as an indicator of the article's emotions. Averages were calculated as this example for pleasantness values:

$$
P=\frac{\sum_{i=1}^{n} W_{i}}{n}
$$

where $P$ represents the average for pleasantness values and $W_{i}$ represents the pleasantness value of the $i$ th word. Averages for attention values, sensitivity values, aptitude values, and polarity values were computed similarly.

\section{Metaphor Analysis}

We analyzed metaphor use for six mental problems and health control based on automatic identified result, including examples of identified metaphors and statistical analysis.

Table 2 shows examples of the most frequently used metaphors for each of seven mental health groups. In order to demonstrate characteristics of each group, we excluded the metaphorical words, which occurred with the most frequency in all mental health groups, such as pay, top, and limit. The same metaphor word was often used in a different way by those in the mentally healthy group compared to those in mental health problem groups, as illustrated in the following examples:

\section{Ex1. Teachers always try their best to meet the re- quirements of students.}

Ex2. We always meet various difficulties on the way to study.

The sentence in the first example was taken from a composition by a student in the healthy control group and expresses a positive sentiment, while the second example was taken from a composition by a student in depression group and expresses a negative sentiment.

We study the emotion of text and effect of metaphor in Student Composition data. The statistical information is shown in Table 3.

Avg. emotion denotes average score of emotion of all text and meta emotion is average emotional score of sentence with metaphor. People in sensitivity group have highest emotional score and that of obsession group is lowest. Meta emotion overall is 0.05 lower than avg. emotion, which shows that, in Student Composition Dataset, students are more likely to express negative emotions and describe sad things through metaphor, for example, sentences $\mathrm{A}$ and $\mathrm{C}$ in Figure 2.

\section{Ex3. My dream was broken \\ Ex4. He will walk into society eventually}

The former expresses the lost mood of broken dream, and the latter is used to show the helpless mood of growing up and entering the society. Both of them apply metaphor to express negative emotions.
To better understand the characteristics of metaphor use for each mental problem, we labeled students as sick or not sick for every particular mental problem and analyzed metaphor features between the two groups. The histograms in Figure 3 show the situation of different metaphor features for each mental health problem. We found that the probability of a sentence containing metaphor was higher among students with inferiority or social phobia than students without these mental problems $(t=1.775, p<0.1 ; t=1.695, p<0.1)$. Students with social phobia were more inclined to use metaphors with negative sentiments than students without social phobia $(t=1.978, p<0.05)$. Additionally, students with obsession had significantly lower scores for average sentiment value of metaphor than students without obsession $(t=-2.060, p<0.05)$. The most distinguishing index of compositions by students with mental health problems was the probability of sentence with metaphor. Students with mental health problems had higher eigenvalues for this variable than those in the healthy group.

\section{Experiments}

We compared the predictive performance of MSM and baseline on the eRisk2017 dataset [49] and on the secondlanguage speaker essays dataset, and we evaluated the metaphor feature with common text features used in baseline. Each of the six mental health problems in the second-language speaker dataset was subjected to a separate bicategorization task. We planned to verify the effectiveness of metaphorical features in the detection of various mental health problems, and we used the same Metaphor-Sentiment feature set in each mental health problem prediction task. Different model parameters will be obtained for different mental problems to deal with metaphorical features.

We applied Synthetic Minority Oversampling Technique (SMOTE) to alleviate the imbalance between positive and negative samples on Student Composition dataset. SMOTE algorithm analyzes samples of minority and produces new samples to the dataset. The specific process: (1) randomly select a sample $x$ from minority and calculate the Euclidean distance between it and other samples in this category; (2) randomly select a sample $x_{n}$ from the $k$ nearest neighbors of $x$ calculated in the previous step; (3) according to the following formula, a new sample is constructed and added to the minority sample set; (4) repeat the above steps until the appropriate sample size is obtained.

$$
x_{\text {new }}=x+\operatorname{rand}(0,1) *\left|x-x_{n}\right| .
$$

5.1. Baseline. The prediction method proposed by [44] was chosen as a baseline since it showed the best performance in eRisk2017 and eRisk2018. They applied two methods to the eRisk2017 dataset to detect people suffering from depression. One method involved logistic regression using features extracted by four word frequency statistics tools-LIWC (http://liwc.wpengine.com/), NRC Emotion Lexicon (http:// www.saifmohammad.com/WebPages/NRC-Emotion-Lexicon. htm), Opinion Lexicon (http://www.cs.uic.edu/ liub/FBS/ opinion-lexicon-English.rar), and VADER Sentiment 
TABLE 2: The examples of frequent metaphors in each state of mind.

\begin{tabular}{lcc}
\hline Mental problem & Frequent metaphor & Example sentence \\
\hline Anxiousness & Hit, present, join & The poor of property can't hit me, but a boring life can \\
Depression & Chase, clean, tough & Maybe there will be many difficulties in the way I chase my dream \\
Inferiority & Support, independent & All these support his spirit of "learning insatiably" \\
Sensitivity & Defeat, move, create & I know in this process some trouble will defeat me \\
Social phobia & Raise, affect, stop & It is really a burden for a poor family to raise a child \\
Obsession & Enter, guide, control & When you enter the society, you probably have problem in finding a job \\
Healthy control & Develop, pass, lead & I want to develop a wonderful game \\
\hline
\end{tabular}

TABLE 3: The statistical information of emotion in various mental states.

\begin{tabular}{lcccccccc}
\hline Mental state & Anxiousness & Depression & Inferiority & Sensitivity & Social Phobia & Obsession & Healthy control & Total \\
\hline Avg. emotion & 0.118 & 0.129 & 0.110 & 0.159 & 0.083 & 0.038 & 0.129 & 0.129 \\
Meta emotion & 0.047 & 0.100 & 0.066 & 0.118 & 0.033 & 0.036 & 0.079 & 0.079 \\
\hline
\end{tabular}

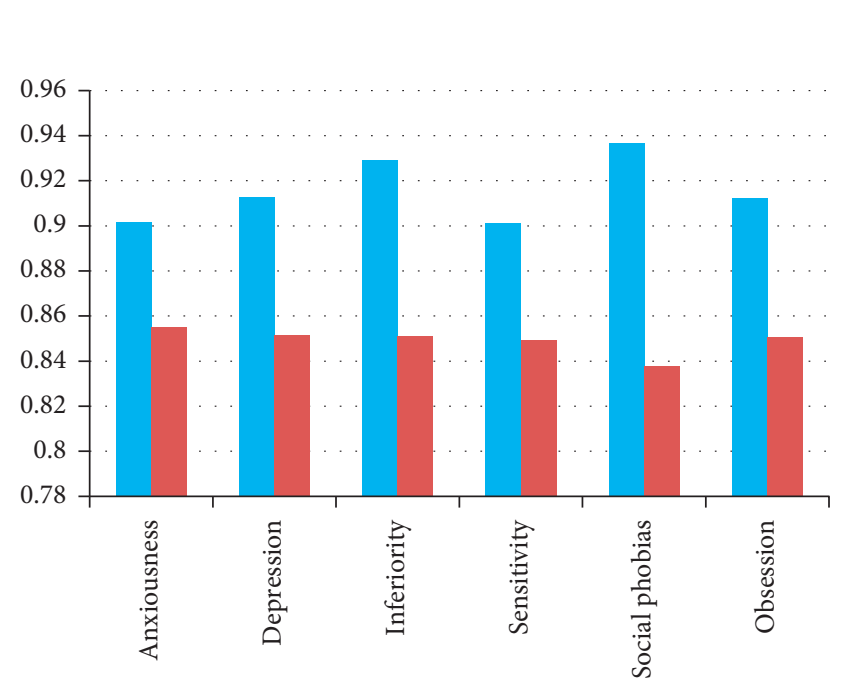

- Patient

- Health control

(a)

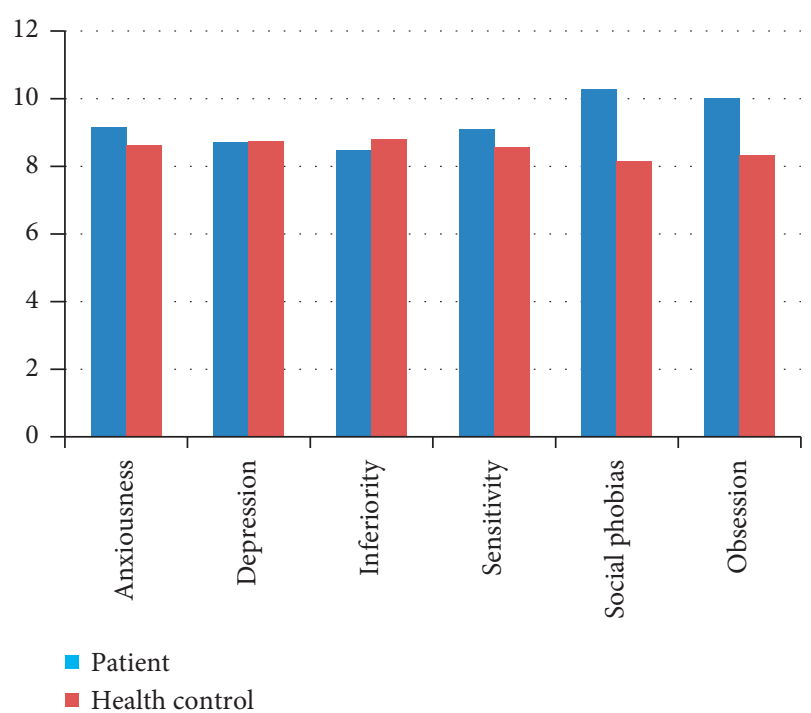

(c)

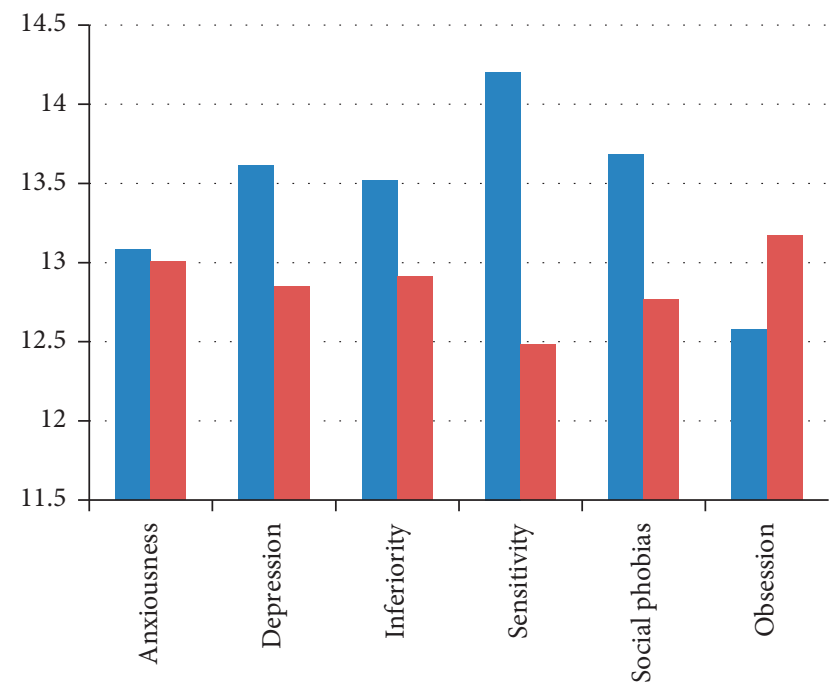

- Patient

- Health control

(b)

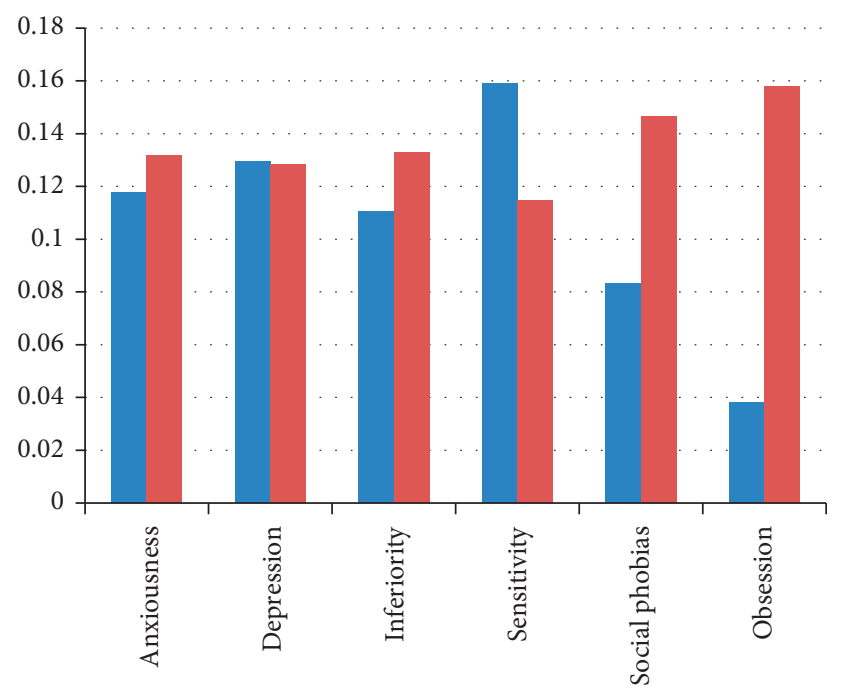

- Patient

- Health control

FIgURE 3: The characteristic of metaphor usage of each mental illness. (a) The probability of sentence with metaphor. (b) The number of positive metaphor. (c) The number of negative metaphor. (d) The average sentiment score of metaphor. 
TABle 4: Prediction performance on the eRisk 2017 dataset.

\begin{tabular}{lccc}
\hline & Method & Accuracy & F1-score \\
\hline \multirow{2}{*}{ Trotzek et al. } & CNN & 0.88 & 0.59 \\
& LR & 0.88 & 0.69 \\
\hline \multirow{3}{*}{ MSM } & Sentiment & 0.81 & 0.61 \\
& Metaphor & 0.87 & 0.56 \\
& ALL & 0.89 & 0.70 \\
\hline
\end{tabular}

All: sentiment + metaphor.

TABle 5: Accuracy of baseline and MSM on six mental illnesses' prediction.

\begin{tabular}{lcccrr}
\hline \multirow{2}{*}{ Method } & \multicolumn{2}{c}{ Trotzek et al. } & & \multicolumn{2}{c}{ MSM } \\
& CNN & LR & All & Sent & 0.61 \\
Anxiousness & 0.75 & 0.71 & $\mathbf{0 . 8 2}$ & 0.63 & 0.71 \\
Depression & 0.73 & 0.69 & 0.75 & 0.80 & 0.70 \\
Inferiority & 0.78 & 0.72 & $\mathbf{0 . 8 0}$ & 0.53 & 0.60 \\
Sensitivity & 0.58 & 0.62 & $\mathbf{0 . 7 1}$ & 0.72 \\
Social phobia & 0.64 & 0.65 & $\mathbf{0 . 7 8}$ & 0.70 \\
Obsession & 0.68 & 0.74 & $\mathbf{0 . 7 8}$ & 0.75 \\
Average & 0.69 & 0.69 & 0.75 \\
\hline
\end{tabular}

Sent: sentiment-based feature set; meta: metaphor-based feature set; all: sent + meta.

TABLE 6: F1-score of baseline and MSM on six mental illnesses' prediction.

\begin{tabular}{lccccc}
\hline \multirow{2}{*}{ Method } & \multicolumn{2}{c}{ Trotzek et al. } & & \multicolumn{2}{c}{ MSM } \\
& CNN & LR & All & Sent & 0.51 \\
\hline Anxiousness & 0.57 & $\mathbf{0 . 6 5}$ & 0.64 & 0.51 & 0.54 \\
Depression & 0.50 & 0.64 & $\mathbf{0 . 6 7}$ & 0.51 & 0.58 \\
Inferiority & 0.46 & 0.63 & 0.62 & 0.44 & 0.50 \\
Sensitivity & 0.46 & 0.59 & 0.73 & 0.50 & 0.70 \\
Social phobia & 0.47 & 0.61 & 0.66 & 0.50 \\
Obsession & 0.42 & $\mathbf{0 . 6 9}$ & $\mathbf{0 . 6 5}$ & 0.59 \\
Average & 0.48 & 0.64 & 0.62 \\
\hline
\end{tabular}

Sent: sentiment-based feature set; meta: metaphor-based feature set; all: sent + meta.

Lexicon (http://www.nltk.org/_modules/nltk/sentiment/vader. html). These tools scan the input text to calculate the frequency of words in different categories, such as the normalized frequency of positive words-words used for expressing positive emotion. The output statistics of word frequency can be transferred into the classifier as text features. The other was a deep learning-based method that employed a convolutional neural network (CNN). We reproduced both methods and compared them with our method for the two datasets.

5.2. Prediction Method. We used a metaphor-based feature set and a sentiment-based feature set to build Metaphor-Sentiment Model (MSM) for predicting mental health status. We compared the performance of three common classifiers: logistic regression, SVM, and neural network. The neural network produced the best results as the relationship between features and mental health problems may be nonlinear. In order to prevent the neural network model from overfitting in training small-scale student dataset, we added L2 regularization, dropout layer, and early-stop mechanism to the model. Meanwhile, the number of layers and hidden layer nodes in the network are determined by testing. 10 -fold cross validation is applied in experiment to ensure the performance of model.

The neural network in this paper was built using Keras (https://github.com/keras-team/keras), which is a fourlayer, fully connected neural network comprising one input layer, two hidden layers, and one output layer. The input layer was the vector that combined the metaphorical features and sentiment features extracted from the data. The two hidden layers had output dimensions of 100 and 50, respectively. The input layer and the two hidden layers used concatenated rectified linear units (CReLU) for the activation function. We added a dropout layer between two hidden layers with a dropout rate of 0.4 to avoid overfitting. The output layer used Softmax for the activation function, which yielded a generalization of logic functions and output vectors with two dimensions.

5.3. Experiment Performance. The eRisk2017 dataset has been divided into a training set and a test set [49]. We tested 


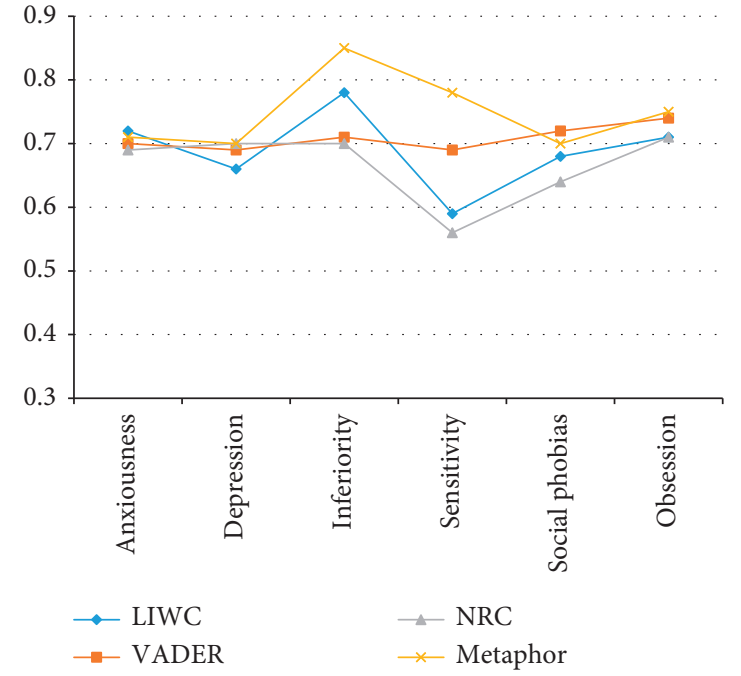

(a)

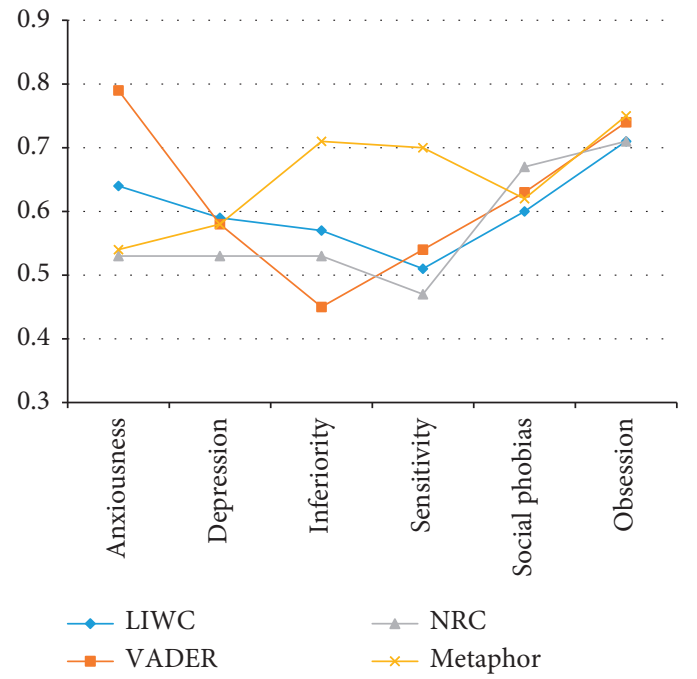

(b)

FIGURE 4: Accuracy and F1-score of every feature separately on six mental illness prediction tasks.

MSM using either a sentiment-based feature set, a metaphor-based feature set, or both and compared the results with those using the baseline method. The results are shown in Table 4. Our identification method outperformed the two baseline methods in terms of both accuracy and F1-score. In addition, the results indicate that metaphor-based feature sets are helpful for detecting depression. These results demonstrate the superiority of our prediction method compared with established methods such as those used for our baseline.

We used 10-fold cross validation to partition our composition dataset collected from second-language students to evaluate the prediction performance of MSM compared to the baseline methods. The results are shown in Tables 5 and 6 .

Table 5 compares the accuracy of the two baseline methods with that of our method for the prediction of six mental health problems. The results show that MSM obtained the highest accuracy, with an average accuracy for all six mental health problems that was significantly higher than baseline (Fisher's exact test: $p<0.05$ ), especially with regard to the sensitivity prediction task (Fisher's exact test: $p<0.005)$. The metaphor-based feature set played an important role in MSM and outperformed the sentiment-based feature set for all mental health group prediction tasks. It achieved the highest accuracy for predicting inferiority, which corresponds to the significant difference in metaphor use between students with inferiority and those without inferiority, as discussed above.

Considering the unbalanced samples, we also computed the F1-score for all mental health problem prediction tasks. The results are shown in Table 6. Overall, using all feature sets, our method showed the highest performance for prediction of the six mental health problems in terms of the average F1score. The improvement in F1-score was significant with respect to students with sensitivity (Fisher's exact test: $p<0.05)$. The logistic regression baseline method achieved the same results as our method overall. The metaphor-based feature set from our method showed the highest F1-scores for predicting inferiority and social phobia.

To further assess the effectiveness of metaphor feature sets, we compared metaphor-based feature set and sentiment-based feature set with three common text features that are extracted by LIWC, NRC Emotion Lexicon, and VADER Sentiment Lexicon and used in the logistic regression baseline method. The line charts shown in Figure 4 present the accuracy and F1-score performance of each feature separately for prediction of the six mental health problems using the neural network classifier. The results show that metaphor feature sets are more effective at predicting inferiority and sensitivity than other textual features and equally effective at predicting other mental health problems.

\section{Conclusions}

To the best of our knowledge, we are the first to demonstrate the prediction of six mental problems-anxiety, depression, inferiority, sensitivity, social phobias, and obsession-using automatically detected metaphors in texts. We used metaphor-based feature sets and sentiment-based feature sets to predict these mental health problems using a compositional dataset produced by second-language students and the eRisk2017 dataset collected from Social Media. Our results show that the proposed method can predict the mental health status of authors of written texts, and our algorithm performs well compared to other state-of-the-art methods. We also analyzed differences in metaphor use among students with various mental health problems and evaluated the effectiveness of metaphor sets compared with other textual features in predicting mental health status from a compositional dataset of second-language students.

Our work demonstrates the value of metaphorical textual features for the prediction of mental health problems. The 
experiment results remind us of the importance of metaphor, as a deep, complex, and cognitive feature for mental health identification, which often focuses on shallow linguistic features. Importantly, we show that metaphor is predictive even for nonnative speakers of the language. We also contribute to a novel, scarce, and valuable dataset, consisting of second-language speakers' essays and data on authors' mental health problems obtained from a psychological survey, which we will release publicly. We hope this paper will stimulate new ideas for the identification and prediction of mental health status through analysis of text and lead to improvement of automated methods for this purpose.

\section{Data Availability}

The data used to support the findings of the study are available from the corresponding author upon request.

\section{Conflicts of Interest}

The authors declare that they have no conflicts of interest.

\section{Acknowledgments}

This work was supported by Social Science Planning Fund Program, Niaoning Province, under grant L20BYY023.

\section{References}

[1] K. Levecque, F. Anseel, A. De Beuckelaer, J. Van der Heyden, and L. Gisle, "Work organization and mental health problems in phD students," Research Policy, vol. 46, no. 4, pp. 868-879, 2017.

[2] R. Bruffaerts, P. Mortier, G. Kiekens et al., "Mental health problems in college freshmen: prevalence and academic functioning," Journal of Affective Disorders, vol. 225, pp. 97103, 2018.

[3] T. M. Evans, L. Bira, J. B. Gastelum, L. T. Weiss, and N. L. Vanderford, "Evidence for a mental health crisis in graduate education," Nature Biotechnology, vol. 36, no. 3, pp. 282-284, 2018.

[4] L. Cameron, Metaphor in Educational Discourse, A\&C Black, London, UK, 2003.

[5] E. Shutova and S. Teufel, "Metaphor corpus annotated for source-target domain mappings," in Proceedings of the International Conference on Language Resources and Evaluation, LREC, vol. 2, Citeseer, Valletta, Malta, May 2010.

[6] D. Zhang, H. Lin, X. Liu, H. Zhang, and S. Zhang, "Combining the attention network and semantic representation for Chinese verb metaphor identification," IEEE Access, vol. 7, pp. 137103-137110, 2019.

[7] P. H. Thibodeau, T. Matlock, and S. J. Flusberg, "The role of metaphor in communication and thought," Language and Linguistics Compass, vol. 13, no. 5, Article ID e12327, 2019.

[8] G. Lakoff and M. Johnson, "Conceptual metaphor in everyday language," The Journal of Philosophy, vol. 77, no. 8, pp. $453-486,1980$.

[9] Z. Kovecses, "Anger: its language, conceptualization, and physiology in the light of cross-cultural evidence," Language and the Cognitive Construal of the World, Cambridge University, Cambridge, UK, pp. 181-196, 1995.
[10] S. Mohammad, E. Shutova, and P. Turney, "Metaphor as a medium for emotion: an empirical study," in Proceedings of the Fifth Joint Conference on Lexical And Computational Semantics, pp. 23-33, Berlin, Germany, August 2016.

[11] V. Dankers, M. Rei, M. Lewis, and E. Shutova, "Modelling the interplay of metaphor and emotion through multitask learning," in Proceedings of the 2019 Conference on Empirical Methods in Natural Language Processing And the 9th International Joint Conference on Natural Language Processing (EMNLP-IJCNLP), pp. 2218-2229, Association of Computational Linguistics, Hong Kong, China, November 2019.

[12] R. M. Billow, J. Rossman, N. Lewis, D. Goldman, and C. Raps, "Observing expressive and deviant language in schizophrenia," Metaphor and Symbol, vol. 12, no. 3, pp. 205-216, 1997.

[13] B. Elvevªg, K. Helsen, M. De Hert, K. Sweers, and G. Storms, "Metaphor interpretation and use: a window into semantics in schizophrenia," Schizophrenia Research, vol. 133, no. 1, pp. 205-211, 2011.

[14] E. D. Gutierrez, G. A. Cecchi, C. Corcoran, and P. Corlett, "Using automated metaphor identification to aid in detection and prediction of first episode schizophrenia," in Proceedings of the 2017 Conference on Empirical Methods in Natural Language Processing, pp. 2923-2930, Copenhagen, Denmark, September 2017.

[15] J. Llewellyn-Beardsley, S. Rennick-Egglestone, F. Callard et al., "Characteristics of mental health recovery narratives: systematic review and narrative synthesis," PLoS One, vol. 14, pp. 1-31, 2019.

[16] D. Magãna, "Cultural competence and metaphor in mental healthcare interactions: a linguistic perspective," Patient Education and Counseling, vol. 102, no. 12, pp. 2192-2198, 2019.

[17] R. Rasetti, V. S. Mattay, L. M. Wiedholz et al., "Evidence that altered amygdala activity in schizophrenia is related to clinical state and not genetic risk," American Journal of Psychiatry, vol. 166, no. 2, pp. 216-225, 2009.

[18] F. M. M. Citron and A. E. Goldberg, "Metaphorical sentences are more emotionally engaging than their literal counterparts," Journal of Cognitive Neuroscience, vol. 26, no. 11, pp. 2585-2595, 2014.

[19] X. Li, M. Zhao, M. Zeng et al., "Hardware Impaired Ambient Backscatter Noma Systems: reliability and security," IEEE Transactions on Communications, vol. 69, no. 4, pp. 27232736, 2021.

[20] L. Sun, L. Wan, and X. Wang, "Learning-based resource allocation strategy for industrial iot in uav-enabled mec systems," IEEE Transactions on Industrial Informatics, vol. 17, no. 7--, pp. 5031-5040, 2020.

[21] L. Sun, J. Wang, and B. Lin, “Task allocation strategy for mecenabled iiots via bayesian network based evolutionary computation," IEEE Transactions on Industrial Informatics, vol. 17, no. 5-, pp. 3441-3449, 2020.

[22] H. Wang, L. Xu, Z. Yan, and T. A. Gulliver, "Low complexity mimo-fbmc sparse channel parameter estimation for industrial big data communications," IEEE Transactions on Industrial Informatics, vol. 17, no. 5-, pp. 3422-3430, 2020.

[23] L. Wan, L. Sun, K. Liu, X. Wang, Q. Lin, and T. Zhu, "Autonomous vehicle source enumeration exploiting non-cooperative UAV in software defined internet of vehicles," IEEE Transactions on Intelligent Transportation Systems, pp. 1-13, 2020.

[24] L. Wan, Y. Sun, L. Sun, Z. Ning, and J. J. P. C. Rodrigues, "Deep learning based autonomous vehicle super resolution doa estimation for safety driving," IEEE Transactions on Intelligent Transportation Systems, 2020. 
[25] L. Xu, H. Wang, and T. A. Gulliver, "Outage probability performance analysis and prediction for mobile iov networks based on ics-bp neural network," IEEE Internet of Things Journal, vol. 8, no. 5-, pp. 3524-3533, 2020.

[26] M. Conway and D. O'Connor, "Social media, big data, and mental health: current advances and ethical implications," Current Opinion in Psychology, vol. 9, pp. 77-82, 2016.

[27] S. Graham, C. Depp, E. E. Lee et al., "Artificial intelligence for mental health and mental illnesses: an overview," Current Psychiatry Reports, vol. 21, no. 11, p. 116, 2019.

[28] S. D'Alfonso, "Ai in mental health," Current Opinion in Psychology, vol. 36, pp. 112-117, 2020.

[29] A. B. R. Shatte, D. M. Hutchinson, and S. J. Teague, "Machine learning in mental health: a scoping review of methods and applications," Psychological Medicine, vol. 49, no. 09, pp. 1426-1448, 2019.

[30] S. Mulyana, S. Hartati, R. Wardoyo, and Subandi, "A processing model using natural language processing (nlp) for narrative text of medical record for producing symptoms of mental disorders," in 2019 Fourth International Conference on Informatics and Computing (ICIC), pp. 1-6, Semarang, Indonesia, October 2019.

[31] M. Levis, C. Leonard Westgate, J. Gui, B. V. Watts, and B. Shiner, "Natural language processing of clinical mental health notes may add predictive value to existing suicide risk models," Psychological Medicine, pp. 1-10, 2020.

[32] H. A. Schwartz, J. Eichstaedt, M. Kern et al., "Towards assessing changes in degree of depression through facebook," in Proceedings of the Workshop on Computational Linguistics and Clinical Psychology: From Linguistic Signal to Clinical Reality, pp. 118-125, University of Cambridge, Cambridge, UK, October 2014.

[33] C. Homan, R. Johar, T. Liu, M. Lytle, V. Silenzio, and C. O. Alm, "Toward macro-insights for suicide prevention: analyzing fine-grained distress at scale," in Proceedings of the Workshop on Computational Linguistics And Clinical Psychology: From Linguistic Signal to Clinical Reality, pp. 107117, Baltimore, MA, USA, June 2014.

[34] G. Coppersmith, M. Dredze, and C. Harman, "Quantifying mental health signals in twitter," in Proceedings of the Workshop on Computational Linguistics and Clinical Psychology: From Linguistic Signal to Clinical Reality, pp. 51-60, Baltimore, MA, USA, June 2014.

[35] R. A. Calvo, D. N. Milne, M. S. Hussain, and H. Christensen, "Natural language processing in mental health applications using non-clinical texts," Natural Language Engineering, vol. 23, no. 5, pp. 649-685, 2017.

[36] T. Nguyen, D. Phung, B. Dao, S. Venkatesh, and M. Berk, "Affective and content analysis of online depression communities," IEEE Transactions on Affective Computing, vol. 5, no. 3, pp. 217-226, 2014.

[37] H.-H. Franco-Penya and L. M. Sanchez, "Text-based experiments for predicting mental health emergencies in online web forum posts," in Proceedings of the Third Workshop on Computational Linguistics And Clinical Psychology, pp. 193197, San Diego, CA, USA, January 2016.

[38] D. N. Milne, G. Pink, B. Hachey, and R. A. Calvo, "Clpsych 2016 shared task: triaging content in online peer-support forums," in Proceedings of the Third Workshop on Computational Linguistics And Clinical Psychology, pp. 118-127, San Diego, CA, USA, June 2016.

[39] A. Cohan, S. Young, and N. Goharian, "Triaging mental health forum posts," in Proceedings of the Third Workshop on
Computational Linguistics And Clinical Psychology, pp. 143147, San Diego, CA, USA, June 2016.

[40] F. Ramiandrisoa, J. Mothe, F. Benamara, and V. Moriceau, "IRIT at eRisk 2018," in Proceedings of the 9th Conference And Labs of the Evaluation Forum, Living Labs (CLEF 2018), pp. 1-12, Avignon, France, September 2018.

[41] D. E. Losada, F. Crestani, and J. Parapar, "Overview of erisk: early risk prediction on the internet," in International Conference of the Cross-Language Evaluation Forum for European Languages, pp. 343-361, Springer, Berlin, Germany, 2018.

[42] J. Weerasinghe, K. Morales, and R. Greenstadt, “"Because... I was told... So much": linguistic indicators of mental health status on twitter," Proceedings on Privacy Enhancing Technologies, vol. 2019, no. 4, pp. 152-171, 2019.

[43] A. Benton, M. Mitchell, and D. Hovy, "Multitask learning for mental health conditions with limited social media data," in Proceedings of the 15th Conference of the European Chapter of the Association for Computational Linguistics: Volume 1, Long Papers, pp. 152-162, Valencia, Spain, April 2017.

[44] M. Trotzek, S. Koitka, and C. M. Friedrich, "Utilizing neural networks and linguistic metadata for early detection of depression indications in text sequences," 2018, http://arxiv.org/ abs/1804.07000.

[45] I. Sekulic and M. Strube, "Adapting deep learning methods for mental health prediction on social media," in Proceedings of the 5th Workshop on Noisy User-Generated Text (W-NUT 2019), Hong Kong, China, November 2019.

[46] L. Tavabi, "Multimodal machine learning for interactive mental health therapy," in 2019 International Conference on Multimodal Interaction, ICMI'19, pp. 453-456, Association for Computing Machinery, New York, NY, USA), October 2019.

[47] Z. Xu, V. P'erez-Rosas, and R. Mihalcea, "Inferring social media users' mental health status from multimodal information," in Proceedings of the 12th Language Resources and Evaluation Conference, pp. 6292-6299, European Language Resources Association, Marseille, France, May 2020.

[48] J. B. Hirsh and J. B. Peterson, "Personality and language use in selfnarratives," Journal of Research in Personality, vol. 43, no. 3, pp. 524-527.

[49] D. E. Losada, F. Crestani, and J. Parapar, "Erisk 2017: clef lab on early risk prediction on the internet: experimental foundations," in Proceedings of the International Conference of the Cross-Language Evaluation Forum for European Languages, pp. 346-360, Springer, Berlin, Germany, August 2017.

[50] D. E. Losada and F. Crestani, "A test collection for research on depression and language use," in Proceedings of the International Conference of the Cross-Language Evaluation Forum for European Languages, pp. 28-39, Springer, Berlin, Germany, August 2016.

[51] J. P. Pestian, P. Matykiewicz, M. Linn-Gust et al., "Sentiment analysis of suicide notes: a shared task," Biomedical Informatics Insights, vol. 5, pp. 1-6, 2012.

[52] S. Gohil, S. Vuik, and A. Darzi, "Sentiment analysis of health care tweets: review of the methods used," JMIR Public Health and Surveillance, vol. 4, no. 2, p. e43, 2018.

[53] R. Mao, C. Lin, and F. Guerin, "Word embedding and wordnet based metaphor identification and interpretation," in Proceedings of the 56th Annual Meeting of the Association for Computational Linguistics (Volume 1: Long Papers), pp. 1222-1231, Melbourne, Australia, July 2018. 